\title{
Some Applications of Statistical Mechanics of Financial Markets
}

\author{
Lester Ingber
}

DRW Investments, LLC

Chicago Mercantile Exchange Center

30 S Wacker Dr Ste 1516

Chicago, IL 60606

and

Lester Ingber Research

PO Box 06440

Wacker Dr PO - Sears Tower

Chicago, IL 60606-0440

\begin{abstract}
Previous development of a statistical mechanics of financial markets (SMFM) is summarized in the context of generalizing a Black-Scholes model of options. Some previously published numerical issues and applications are highlighted.
\end{abstract}

\section{Standard Black-Scholes (BS) Model}

The standard partial-differential equation used to formulate most variants of Black-Scholes (BS) models describing the market value of an option, $V$, is

$$
\frac{\partial V}{\partial t}+\frac{1}{2} \sigma^{2} S^{2} \frac{\partial^{2} V}{\partial S^{2}}+r S \frac{\partial V}{\partial S}-r V=0
$$

where $S$ is the asset price, and $\sigma$ is the standard deviation, or volatility of $S$, and $r$ is the short-term interest rate. The solution depends on boundary conditions, subject to a number of interpretations, some requiring minor transformations of the basic BS equation or its solution. For example, the basic equation can apply to a number of one-dimensional models of interpretations of prices given to $V$, e.g., puts or calls, and to $S$, e.g., stocks or futures, dividends, etc.

For instance, if $V$ is set to $C$, a call on an European option with exercise price $E$ with maturity at $T$, the solution is

$$
\begin{gathered}
C(S, t)=S N\left(d_{1}\right)-E e^{-r(T-t)} N\left(d_{2}\right), \\
d_{1}=\frac{\ln (S / E)+\left(r+\frac{1}{2} \sigma^{2}\right)(T-t)}{\sigma(T-t)^{1 / 2}} \\
d_{2}=\frac{\ln (S / E)+\left(r-\frac{1}{2} \sigma^{2}\right)(T-t)}{\sigma(T-t)^{1 / 2}} .
\end{gathered}
$$

\subsection{Some Key Issues in Derivation of BS}

The basic BS model considers a portfolio in terms of delta $(\Delta)$,

$$
\Pi=V-\Delta S
$$

in a market with Gaussian-Markovian ("white") noise $X$ and drift $\mu$,

$$
\frac{d S}{S}=\sigma d X+\mu d t
$$

where $V(S, t)$ inherits a random process from $S$, 


$$
d V=\sigma S \frac{\partial V}{\partial S} d X+\left(\mu S \frac{\partial V}{\partial S}+\frac{1}{2} \sigma^{2} S^{2} \frac{\partial^{2} V}{\partial S^{2}}+\frac{\partial V}{\partial t}\right) d t
$$

This yields

$$
d \Pi=\sigma\left(\frac{\partial V}{\partial S}-\Delta\right) d X+\left(\mu S \frac{\partial V}{\partial S}+\frac{1}{2} \sigma^{2} S^{2} \frac{\partial^{2} V}{\partial S^{2}}+\frac{\partial V}{\partial t}-\mu \Delta S\right) d t
$$

The expected risk-neutral return of $\Pi$ is

$$
d \Pi=r \Pi d t=r(V-\Delta S) d t .
$$

Options $V$ on futures $F$ can be derived, e.g., using simple transformations such as

$$
F=S e^{r(T-t)},
$$

and setting

$$
d \Pi=r V d t .
$$

The corresponding BS equation for futures $F$ is

$$
\frac{\partial V}{\partial t}+\frac{1}{2} \sigma^{2} F^{2} \frac{\partial^{2} V}{\partial S^{2}}-r V=0 .
$$

At least two advantages are present if $\Delta$ is chosen such that

$$
\Delta=\frac{\partial V}{\partial S} \text {. }
$$

Then, the portfolio can be instantaneously "risk-neutral," in terms of zeroing the coefficient of $X$, as well as independent of the direction of market, in terms of zeroing the coefficient of $\mu$. For the above example of $V=C$,

$$
\Delta=N\left(d_{1}\right) \text {. }
$$

Other trading strategies based on this simple model are based on hedging with other similar constructs, e.g., using gamma $(\Gamma)$, theta $(\Theta)$, vega, rho $(\rho)[1]$,

$$
\begin{aligned}
& \Gamma=\frac{\partial^{2} \Pi}{\partial S^{2}}, \\
& \Theta=\frac{\partial \Pi}{\partial t}, \\
& \text { vega }=\frac{\partial \Pi}{\partial \sigma}, \\
& \rho=\frac{\partial \Pi}{\partial r} .
\end{aligned}
$$

The BS equation, Eq. (1), may be written as

$$
\Theta+r S \Delta+\frac{1}{2}(\sigma S)^{2} \Gamma=r f .
$$

\section{Time Dependent $\sigma(t)$ and $r(t)$}

The volatility $\sigma$ may depend on other variables, and the BS model may be generalized to multivariable models. However, within the framework of the basic BS model, if $\sigma$ and $r$ are timedependent, then it turns out that the above solutions of the basic BS, and the use of the above set of 
$\{\Delta, \Gamma, \Theta$, vega, $\rho\}$, etc., can be used without change, provided an "effective" volatility, $\hat{\sigma}$, is defined in terms of $\sigma(t)$, and an "effective" interest-rate, $\hat{r}$, is defined in terms of $r(t)$, are defined.

This can be developed by considering a slight generalization of the above BS equation for the variable $\hat{V}$, using methods given in a standard text [2]. A transformation of variables is used to transform away any time-dependent coefficients,

$$
\begin{aligned}
& \hat{S}=S e^{\alpha(t)}, \\
& \hat{V}=V e^{\beta(t)}, \\
& \hat{t}=\gamma(t) .
\end{aligned}
$$

This leads to

$$
\frac{d \gamma(t)}{d t} \frac{\partial \hat{V}}{\partial \hat{t}}+\frac{1}{2} \sigma(t)^{2} \hat{S}^{2} \frac{\partial^{2} \hat{V}}{\partial \hat{S}^{2}}+\left(r(t)+\frac{d \alpha(t)}{d t}\right) \hat{S} \frac{\partial \hat{V}}{\partial \hat{S}}-\left(r(t)+\frac{d \beta(t)}{d t}\right) \hat{V}=0 .
$$

Taking

$$
\begin{aligned}
& \alpha(t)=\int_{t}^{T} r(\tau) d \tau, \\
& \beta(t)=\int_{t}^{T} r(\tau) d \tau, \\
& \gamma(t)=\int_{t}^{T} \sigma^{2}(\tau) d \tau,
\end{aligned}
$$

leads to

$$
\frac{\partial \hat{V}}{\partial \hat{t}}=\frac{1}{2} \hat{S}^{2} \frac{\partial^{2} \hat{V}}{\partial \hat{S}^{2}}
$$

with coefficients independent of time $t$ (the motivation for this transformation). In terms of $V$,

$$
V(S, t)=e^{-\beta(t)} \hat{V}\left(S e^{\alpha(t)}, \gamma(t)\right) .
$$

To get the explicit form of the solution, consider the original BS model with constant coefficients and solution $V_{B S}$ :

$$
\left.V_{B S}=e^{-(T-t) r} \hat{V}_{B S}\left(S e^{-(T-t) r},(T-t) \sigma^{2}\right)\right),
$$

for some solution $\hat{V}_{B S}$. Therefore, the standard BS solutions for various products can be used if $\sigma$ and/or $r$ are time-dependent, by replacing $\sigma$ in the original equation by $\hat{\sigma}$, and by replacing $r$ in the original equation by $\hat{r}$,

$$
\begin{aligned}
& \sigma^{2} \rightarrow \hat{\sigma}^{2}=\frac{1}{T-t} \int_{t}^{T} \sigma^{2}(\tau) d \tau, \\
& r \rightarrow \hat{r}=\frac{1}{T-t} \int_{t}^{T} r(\tau) d \tau .
\end{aligned}
$$




\subsection{Example of Use}

This can be very useful in conjunction with models of time-dependent volatilities and interest-rates. For example, if volatilities are fit to data, and a moving-average optimization gives a form like

$$
\sigma(t)=A(T-t)^{z},
$$

then the effective $\hat{\sigma}$ to use for the day's trading would be the scaled $\sigma(t)$,

$$
\hat{\sigma}=\frac{A(T-t)^{z}}{\sqrt{2 z+1}}=\frac{\sigma(t)}{\sqrt{2 z+1}} .
$$

\section{Some Discretization Issues}

Since tree approximations and/or discretization of variables are often applied to calculating the above entities, it is useful to apply limits to some of these discretizations wherever possible [3-5].

Eq. (1) has another mathematically equivalent path-integral representation [6], which often offers some advantages in formulation, calculation, and inclusion of boundary conditions. Here, attention is drawn to the derivation of limits on the meshes of $\delta t$ and $\delta V$ such that there can be maintained numerical equivalence between these equivalent algebraic representations.

\subsection{Path-Integral Representation}

The short-time conditional representation of $V$ in an epoch $\delta t$ is given in terms of the "Lagrangian" $L$ in the Itô prepoint discretization,

$$
\begin{aligned}
& V[S, t+\delta t \mid S, t]=\left(2 \pi(\sigma S)^{2} \delta t\right)^{-1 / 2} \exp (-L \delta t), \\
& L=\frac{(\dot{S}+r S)^{2}}{2(\sigma S)^{2}}+r, \\
& \dot{S}=\frac{\delta S}{\delta t}=\frac{S(t+\delta t)-S(t)}{\delta t} .
\end{aligned}
$$

For Eq. (1), the condition on the mesh of $\delta t$ is that it be no coarser than

$$
\delta t \leq \frac{2(\sigma S)^{2}}{(r S)^{2}}=\frac{2 \sigma^{2}}{r^{2}}
$$

throughout the ranges of $S$ giving the most important contributions to $V$, e.g., where $\dot{S}$ is small. The mesh of $S$ is optimally chosen such that $\delta S$ is measured by the variance $(\sigma S)^{2}$,

$$
\delta S \approx \sigma S(\delta t)^{1 / 2} .
$$

For Eq. (10), similarly, the mesh of $F$ is measured by the variance,

$$
\delta F \approx \sigma F(\delta t)^{1 / 2} \text {. }
$$

However, here there is no interest-rate drift term, the above criteria for $\delta t$ is not applicable, and so

$$
\delta t \leq \frac{2(\sigma F)^{2}}{\dot{F}^{2}}
$$

for some "typical" $\dot{F}$.

Note that if $\sigma$ and $r$ are time-dependent, then, as discussed above, $\hat{\sigma}$ and $\hat{r}$ are the appropriate variables to use in these constraints on the meshes. Also, in the region of boundary and final conditions, often tighter meshes are required.

\subsection{Itô Representation Transformations}

Some care must be taken with nonconstant drifts and diffusions. For example, for purposes of calculating volatilities, it is often convenient to transform to a variable $Z$ 


$$
Z=\ln S \text {. }
$$

The above distribution can be transformed into $V[Z, t+\delta t \mid Z, t]$,

$$
\begin{array}{r}
d S_{t} V[S, t+\delta t \mid S, t]=d Z_{t} V[Z, t+\delta t \mid Z, t] \\
=d Z_{t}\left(2 \pi \sigma^{2} \delta t\right)^{-1 / 2} \exp \left(-L^{\prime} \delta t\right), \\
L^{\prime} \delta t=\frac{\left(\left[\exp \left(Z_{t+\delta t}-Z_{t}\right)-1\right]+r\right)^{2}}{2 \sigma^{2} \delta t}+r \delta t .
\end{array}
$$

This can be expanded into

$$
\begin{aligned}
& L^{\prime} \delta t \approx \frac{\left.\left(Z_{t+\delta t}-Z_{t}+\frac{1}{2}\left(Z_{t+\delta t}-Z_{t}\right)^{2}-r \delta t\right)\right)^{2}}{2 \sigma^{2} \delta t}+r \delta t \\
& \approx \frac{\left(Z_{t+\delta t}-Z_{t}-\left(r-\frac{1}{2} \sigma^{2}\right) \delta t\right)^{2}}{2 \sigma^{2} \delta t}+r \delta t, \\
&\left(Z_{t+\delta t}-Z_{t}\right)^{2} \approx \sigma^{2} \delta t,
\end{aligned}
$$

where only terms of order $\delta t$ have been kept, yielding

$$
L^{\prime}=\frac{\left(\dot{Z}-\left(r-\frac{1}{2} \sigma^{2}\right)\right)^{2}}{2 \sigma^{2}}+r .
$$

This defines the distribution $V[Z, t+\delta t \mid Z, t]$ in terms of the distribution $V[S, t+\delta t \mid S, t]$, where the volatilities differ by a factor of $S^{-1}$ and the drifts differ by the term $-\frac{1}{2} \sigma^{2}$. The above procedure is valid in the multivariate case for more general nonlinear drifts and diffusions to order $\delta t^{3 / 2}$ [6].

\section{Nonlinear Nonequilibrium Markets}

It is clear that during very volatile markets, the BS formalism is both not as applicable nor is it as practically applicable (orders can't get filled, markets close, etc.) as during relatively "normal" market conditions.

In such highly nonlinear nonequilibrium contexts, it may be useful to look for other criteria that can maximize profits and reduce risk. A formal approach to a statistical mechanics of nonlinear financial markets (SMFM) [7] has been shown to be useful in other disciplines, e.g., analyses of electroencephalography and combat scenarios, as documented in several papers available from the http://www.ingber.com archive. The development of powerful generic numerical techniques, e.g., adaptive simulated annealing (ASA) optimization [8] and path-integral (PATHINT) calculations have been demonstrated to be give numerical support to the sophisticated SMFM algebra, in general to the several disciplines just mentioned, and specifically to financial markets [9-12]. This formalism may be useful as well for nonlinear nonequilibrium derivatives, e.g., by developing general "Euler-Lagrange" equations of motion and canonical momenta indicators (CMI) of multivariate markets.

\subsection{Generalized BS Model}

For example, consider a plausible generalization of the basic stochastic equations above defining a stock and its option,

$$
\frac{d S}{S}=\sigma d X+\mu d t, d S=\sigma S d X+\left(\mu-\frac{1}{2} \sigma^{2}\right) S d t
$$




$$
d V=v d Y+\sigma S \frac{\partial V}{\partial S} d X+\left(\mu S \frac{\partial V}{\partial S}+\frac{1}{2} \sigma^{2} S^{2} \frac{\partial^{2} V}{\partial S^{2}}+\frac{\partial V}{\partial t}\right) d t
$$

where an independent random process $d Y$ with coefficient $v$ has been added to the option, which is plausible under volatile options trading. The coefficient $v$ may itself be a function,

$$
v=v(V, S, t) \text {. }
$$

A reasonable argument can be given for some degree of independent noise in an option addition to that derived from its underlying security. It is now widely accepted that volatility of traded securities is primarily incurred during actual trading days, e.g., 252 days/year, such that yearly rates of volatility are scaled accordingly $[13,14]$. Put another way, the activity of trading may induce volatility in any given security, based in large part on how traders perceive the market. It is not uncommon for example to find relatively high implied volatilities of options on relatively low volatilities of their underlying securities.

The above path-integral representation of this two-variable system is given by the probability distribution $P$, in a prepoint discretized representation,

$$
\begin{aligned}
& P[S, V ; t+\delta t \mid S, V ; t]=\left(2 \pi \delta t D^{1 / 2}\right)^{-1} \exp (-L \delta t), \\
& D=(\sigma S)^{4}\left(1+\varepsilon^{2}-\Delta^{2}\right), \\
& \varepsilon=\frac{v}{\sigma S \Delta}, \\
& L=\frac{1}{(\sigma S)^{2}}\left[\frac{\left(\dot{S}-\left(\mu-\frac{1}{2} \sigma^{2}\right) S\right)^{2}}{2}+\frac{\left(\dot{S}-\left(\mu-\frac{1}{2} \sigma^{2}\right) S\right)(\dot{V}-\beta)}{\Delta}+\frac{(\dot{V}-\beta)^{2}}{2\left(1+\varepsilon^{2}\right)}\right], \\
& \beta=\left(\mu-\frac{1}{2} \sigma^{2}\right) S \Delta+\Theta+\frac{1}{2}(\sigma S)^{2} \Gamma .
\end{aligned}
$$

where the intent is to use empirical data for $\Delta, \Theta$, and $\Gamma$.

\subsection{Euler-Lagrange (EL) Equations}

The above two-variable set of stochastic differential equations for $S$ and $V$ of course can be processed by the BS methodology, subject to linear algebraic constraints, essentially combining weighted sums of these equations to subtract out the noise terms in $d X$ and $d Y$ to contribute to a "risk-neutral" portfolio [1].

However, there exist other techniques to constrain such systems to paths that are not affected by "noise" to at least first and second order. For example, consider that the variational principle for $L$ possessed by this probability distribution $P, \delta L=0$, leads to Euler-Lagrange (EL) coupled second-order (in $t$ ) ordinary differential equations (given below for a more general multivariate system). These equations can possess "steady-state" solutions, which are "deterministic" in that they "ride" over the stochastic variables of the system.

In other words, the EL equations can lead to values of $\{\Delta, \Gamma, \Theta$, vega, $\rho\}$ useful for "risk-neutral" hedging that are different from those obtained by the BS model. (Some economists would argue that additionally $\mu$ be set to $r$ to have these equations enforce risk-neutral hedging.) These new solutions do not have as restrictive underlying assumptions, and they may be useful especially in highly volatile markets.

\subsection{Discretizations and Riemannian geometry}

Coupling of dependences of other markets are included in a straight-forward manner in the above formalism, and their parameters are readily optimized using ASA. It is convenient to use a more general compact notation for this purpose. For example, in terms of variables labeled by $G$, with drifts $f^{G}$ and 
metric $g_{G G^{\prime}}$ (the inverse of the covariance matrix $g^{G G^{\prime}}$ ),

$$
\begin{aligned}
M^{G} & =\left(\begin{array}{l}
S \\
V
\end{array}\right), \\
f^{G} & =\left(\begin{array}{c}
\mu S \\
\beta
\end{array}\right), \\
\hat{g}_{j}^{G} & =\left(\begin{array}{ll}
\sigma S & 0 \\
\sigma S & v
\end{array}\right), \\
g^{G G^{\prime}} & =\hat{g}_{j}^{G} \hat{g}_{j}^{G^{\prime}} \\
& =(\sigma S)^{2}\left(\begin{array}{rr}
1 & \Delta \\
\Delta & \left(1+\varepsilon^{2}\right)
\end{array}\right) .
\end{aligned}
$$

In this notation, the Itô stochastic equations for $S$ and $V$ are

$$
\dot{M}^{G}=f^{G}+\hat{g}_{j}^{G} \eta^{j},
$$

where the Einstein summation convention is used, wherein factors with repeated indices are summed over. The Lagrangian in the prepoint discretized representation is given as

$$
\begin{aligned}
& L=\frac{1}{2}\left(\dot{M}^{G}-g^{G}\right) g_{G G^{\prime}}\left(\dot{M}^{G^{\prime}}-g^{G^{\prime}}\right)-\Phi, \\
& g^{G}=f^{G},
\end{aligned}
$$

where $\Phi$ is an additional "potential" term, here zero.

Note that if the above stochastic differential equations were written in the Stratonovich midpoint discretization, then we would have calculated

$$
\begin{gathered}
g^{G}=f^{G}+\frac{1}{2} \hat{g}_{j}^{G^{\prime}} \hat{g}_{j, G^{\prime}}^{G} \\
=\left(\begin{array}{c}
\mu S \\
\beta
\end{array}\right), \\
{[\cdots]_{, G}=\frac{\partial[\cdots]}{\partial G} .}
\end{gathered}
$$

The one-dimensional BS Eq. (1) can be written similarly, where $V$ is the short-time conditional "probability," only $M^{G}=S$ exists in $L$, and $\Phi=-r$.

There are of course other discretizations than the Itô prepoint discretization of stochastic differential equations such as Eq. (4), related to other differential-operator orderings of partial differential equations such as Eq. (1), also related to other transformations of variables in Lagrangians such as Eq. (23) [6]. This can be understood in the Stratonovich (midpoint discretized) representation for more than two variables for nonconstant diffusions, where it is seen that a Riemannian geometry is induced by the metric $g_{G G^{\prime}}$, exhibiting the invariance of the basic probability distribution under this geometry [7].

For example, the Lagrangian in in Eq. (23) is in the Itô prepoint representation. In the Stratonovich midpoint discretization, the path integral for the long-time evolution of the probability distribution in terms of multiple foldings of the short-time distributions is

$$
P\left[M_{t} \mid M_{t_{0}}\right] d M(t)=\int \cdots \int \underline{D} M \exp (-S) \delta\left[M\left(t_{0}\right)=M_{0}\right] \delta\left[M(t)=M_{t}\right],
$$




$$
\begin{aligned}
& S=\min \int_{t_{0}}^{t} d t^{\prime} L, \\
& \underline{D} M=\lim _{u \rightarrow \infty} \prod_{\rho=1}^{u+1} g^{1 / 2} \prod_{G}(2 \pi \delta t)^{-1 / 2} d M_{\rho}^{G}, \\
& L\left(\dot{M}^{G}, M^{G}, t\right)=\frac{1}{2}\left(\dot{M}^{G}-h^{G}\right) g_{G G^{\prime}}\left(\dot{M}^{G^{\prime}}-h^{G^{\prime}}\right)+\frac{1}{2} h_{; G}^{G}+R / 6-\Phi, \\
& h^{G}=g^{G}-\frac{1}{2} g^{-1 / 2}\left(g^{1 / 2} g^{G G^{\prime}}\right)_{G^{\prime}}, \\
& g_{G G^{\prime}}=\left(g^{G G^{\prime}}\right)^{-1}, \\
& g^{\prime}=\operatorname{det}\left(g_{G G^{\prime}}\right), \\
& h_{; G}^{G}=h_{, G}^{G}+\Gamma_{G F}^{F} h^{G}=g^{-1 / 2}\left(g^{1 / 2} h^{G}\right)_{, G}, \\
& \Gamma_{J K}^{F} \equiv g^{L F}[J K, L]=g^{L F}\left(g_{J L, K}+g_{K L, J}-g_{J K, L}\right), \\
& R=g^{J L} R_{J L}=g^{J L} g^{J K} R_{F J K L}, \\
& R_{F J K L}=\frac{1}{2}\left(g_{F K, J L}-g_{J K, F L}-g_{F L, J K}+g_{J L, F K}\right)+g_{M N}\left(\Gamma_{F K}^{M} \Gamma_{J L}^{N}-\Gamma_{F L}^{M} \Gamma_{J K}^{N}\right) .
\end{aligned}
$$

(Some authors use $g=\operatorname{det}\left(g^{G G^{\prime}}\right)$, especially when is it convenient to identify $g$ with $\sigma^{2}$ in the limit of one variable.)

\subsection{Most Probable Transitions}

Another set of coupled equations that can be useful if only most probable transition states are sought, are simple coupled first-order rate equations [15],

$$
\dot{M}^{G}=f^{G}-\frac{1}{2} g^{1 / 2}\left(g^{-1 / 2} g^{G G^{\prime}}\right)_{G^{\prime}} .
$$

\subsection{Canonical Momenta Indicators (CMI)}

The output of all this algebra need not be confined to complex algebraic forms or tables of numbers. Because $L$ possesses a variational principle, sets of contour graphs, at different long-time epochs of the path-integral of $P$ over its variables at all intermediate times, give a visually intuitive and accurate decision-aid to view the dynamic evolution of the scenario.

For example, this Lagrangian approach permits a quantitative assessment of concepts usually only loosely defined,

$$
\begin{aligned}
& \text { "Momentum" }=\Pi^{G}=\frac{\partial L}{\partial\left(\partial M^{G} / \partial t\right)}, \\
& \text { "Mass" } g_{G G^{\prime}}=\frac{\partial^{2} L}{\partial\left(\partial M^{G} / \partial t\right) \partial\left(\partial M^{G^{\prime}} / \partial t\right)}, \\
& \text { "Force" }=\frac{\partial L}{\partial M^{G}}, \\
& \text { “F=ma": } \delta L=0=\frac{\partial L}{\partial M^{G}}-\frac{\partial}{\partial t} \frac{\partial L}{\partial\left(\partial M^{G} / \partial t\right)},
\end{aligned}
$$


where the last " $F=m a$ " equation is the set of EL equations discussed above. These physical entities provide another form of intuitive, but quantitatively precise, presentation of these analyses. For example, daily newspapers use this terminology to discuss the movement of security prices.

The CMI can be used to develop trading rules [12]. The extreme sensitivity of the CMI gives rapid feedback on the changes in trends as well as the volatility of markets. A time-locked moving average, yielding averages over each time in moving windows during which the model is fit to data, provides manageable indicators for trading signals. 


\section{References}

1. J.C. Hull, Options, Futures, and Other Derivatives, Third Edition, Prentice Hall, Upper Saddle River, NJ, (1997).

2. P. Wilmott, S. Howison, and J. Dewynne, The Mathematics of Financial Derivatives, Cambridge U Press, Cambridge, (1995).

3. M.F. Wehner and W.G. Wolfer, Numerical evaluation of path-integral solutions to Fokker-Planck equations. I., Phys. Rev. A 27, 2663-2670 (1983).

4. L. Ingber, H. Fujio, and M.F. Wehner, Mathematical comparison of combat computer models to exercise data, Mathl. Comput. Modelling 15 (1), 65-90 (1991).

5. L. Ingber, Statistical mechanics of neocortical interactions: Path-integral evolution of short-term memory, Phys. Rev. E 49 (5B), $4652-4664$ (1994).

6. F. Langouche, D. Roekaerts, and E. Tirapegui, Functional Integration and Semiclassical Expansions, Reidel, Dordrecht, The Netherlands, (1982).

7. L. Ingber, Statistical mechanics of nonlinear nonequilibrium financial markets, Math. Modelling 5 (6), 343-361 (1984).

8. L. Ingber, Adaptive Simulated Annealing (ASA), Global optimization C-code, Lester Ingber Research, Chicago, IL, (1993).

9. L. Ingber, Statistical mechanical aids to calculating term structure models, Phys. Rev. A 42 (12), 7057-7064 (1990).

10. L. Ingber, M.F. Wehner, G.M. Jabbour, and T.M. Barnhill, Application of statistical mechanics methodology to term-structure bond-pricing models, Mathl. Comput. Modelling 15 (11), 77-98 (1991).

11. L. Ingber, Statistical mechanics of nonlinear nonequilibrium financial markets: Applications to optimized trading, Mathl. Computer Modelling 23 (7), 101-121 (1996).

12. L. Ingber, Canonical momenta indicators of financial markets and neocortical EEG, in Progress in Neural Information Processing, (Edited by S.-I. Amari, L. Xu, I. King, and K.-S. Leung), pp. 777-784, Springer, New York, (1996).

13. E.E. Fama, The behavior of stock market prices, J. Business 38, 34-105 (1965).

14. K.R. French, Stock returns and the weekend effect, J. Finan. Econ. 8, 55-69 (1980).

15. H. Dekker, On the most probable transition path of a general diffusion process, Phys. Lett. A 80, 99-101 (1980). 